\section{La Révolution française}

Cahiers de l'Institut d'histoire de la Révolution française

$16 \mid 2019$

1801-1840 - Haïti, entre Indépendance et

Restauration

\title{
L'Empire français et la langue italienne : le débat sur la langue et les conséquences de l'époque napoléonienne en Italie
}

\section{Elisa Baccini}

\section{OpenEdition}

\section{Journals}

Édition électronique

URL : http://journals.openedition.org/lrf/2680

DOI : $10.4000 /$ Irf.2680

ISSN : 2105-2557

\section{Éditeur}

IHMC - Institut d'histoire moderne et contemporaine (UMR 8066)

Référence électronique

Elisa Baccini, « L’Empire français et la langue italienne : le débat sur la langue et les conséquences de l'époque napoléonienne en Italie ", La Révolution française [En ligne], 16 | 2019, mis en ligne le 20 juin 2019, consulté le 22 juin 2019. URL : http://journals.openedition.org//rf/2680 ; DOI : 10.4000/Irf.2680

Ce document a été généré automatiquement le 22 juin 2019

(c) La Révolution française 


\title{
L'Empire français et la langue italienne : le débat sur la langue et les conséquences de l'époque napoléonienne en Italie
}

\author{
Elisa Baccini
}

1 Paul Hazard, dans sa thèse de doctorat de 1910, La Révolution française et les Lettres italiennes, a présenté les résultats d'une étude qui analysait en détail le débat sur la présence française en Italie et ses conséquences sur la langue italienne ${ }^{1}$. Dès le titre, il annonçait son intention de mettre l'accent sur les liens entre l'époque française et le monde littéraire de l'Italie. Il voulait prouver que l'époque napoléonienne avait marqué la fin de la longue influence de la culture et de la langue française en Italie. Le travail de Paul Hazard était, en fait, basé sur une réflexion qui peut se résumer par la question suivante: en supposant qu'en 1789 l'hégémonie culturelle et linguistique de la France était incontestée en Italie, comment expliquer la fin de cette hégémonie et le fait que la défaite de Napoléon en Italie a marqué le triomphe de l'autonomie littéraire du pays ? À cette question Hazard répondait que la conquête française avait déclenché une réaction qui s'exprima non seulement dans la littérature, mais aussi dans toute la société italienne. En France même, les mouvements littéraires nationaux avaient rencontré des concurrents inattendus venus d'Allemagne. C'est leur influence qui a aussi servi en Italie pour combattre les Français et pour affirmer l'autonomie intellectuelle du pays.

2 Cependant, la multitude des thèmes de recherche suggérés par Paul Hazard et les nombreuses idées qu'il avait proposées n'ont guère trouvé d'écho dans le milieu universitaire italien notamment quant au développement, pourtant crucial, d'une réflexion sur les effets de la domination française sur la langue italienne. Bien qu'en France le travail de Hazard ait été très apprécié et lui ait finalement valu une élection au collège de France en $1925^{2}$, il reçut en Italie un accueil mitigé ${ }^{3}$. Certains ont applaudi sa thèse pour sa finesse et la richesse de ses sources. D'autres ont observé qu'elle présentait, dans un moment délicat de l'histoire nationale italienne, le énième spectacle de la 
prééminence française sur la culture européenne et italienne. À cet égard, la recension écrite par Giovanni Gentile en 1911 dans le périodique dirigé par Benedetto Croce, La Critica, est intéressante ${ }^{4}$. Gentile reconnaît la richesse des recherches archivistiques et bibliographiques du travail de Hazard, mais rejette clairement la thèse de la dépendance de la culture littéraire italienne vis-à-vis de la France, notamment dans les conséquences sur le Risorgimento ${ }^{5}$. C'est peut-être la raison pour laquelle la traduction du texte de Hazard en italien n'a été publiée qu'en 1995, soit 85 ans après son apparition en France ${ }^{6}$.

Par conséquent, pour tenter de faire vivre l'héritage de Paul Hazard et dans le cadre d'une plus large recherche, cet article vise à relancer certains questionnements cruciaux pour comprendre les effets de la période napoléonienne en Italie, mais également pour mettre en évidence la relation entre l'histoire de la langue et le processus de construction de la nation italienne. Celui-ci a pris une forme concrète seulement après la chute de Napoléon, mais pendant l'occupation française, il s'est nourri de la présence étrangère. Il s'agira ainsi de comprendre comment les politiques linguistiques s'insèrent dans le cadre idéologique de l'Empire napoléonien. À cet égard, Hazard soulignait déjà dans sa thèse l'attitude de supériorité et la volonté de domination que le gouvernement napoléonien assumait envers les lettres, la langue et la culture en Italie. Cet infranciosamento des coutumes et de la langue n'est pas le simple résultat de l'influence exercée par les Français, mais provient en partie également d'une politique imposant aux sujets la culture de leurs souverains, c'est à dire une forme d'impérialisme culturel ${ }^{7}$. Cependant, dans les paragraphes suivants, il apparaitra que Napoléon n'a pas toujours suivi une ligne politique très ferme.

\section{Le débat sur la langue}

4 Tout d'abord, comme mentionné dans les considérations de Hazard, l'influence de la langue française sur la langue italienne est apparue bien avant la Révolution française et la parenthèse napoléonienne qui a suivi. Peu de temps après la naissance de la soi-disante questione della lingua, une formule qui condense le débat pluriséculaire sur la formation et la définition de la langue italienne, il y avait déjà des traces de l'influence du français sur l'italien ${ }^{8}$. C'est cependant au cours du XVIII ${ }^{\mathrm{e}}$ siècle que la discussion prend brusquement une autre tournure, tout comme l'italien.

5 «Quand une langue change rapidement, c'est le signe sûr d'une révolution dans les idées de la nation ${ }^{9} »$ : dans cette phrase, Cesare Beccaria n'a pas seulement brièvement résumé le lien étroit entre les idées et la langue d'une nation. En effet, lorsqu'il écrit en 1764, la culture des Lumières est au sommet de sa diffusion en Italie et les intellectuels les plus ouverts essaient de s'en imprégner le plus rapidement et le plus largement possible. La révolution des idées, dont parle Beccaria, implique toute la civilisation littéraire italienne, ainsi que la langue. À l'époque, la pénétration des idées des Lumières et de la langue française dans l'esprit des intellectuels contemporains avait en effet provoqué un changement décisif et radical dans le débat linguistique. À cet égard, les écrivains milanais du périodique Il Caffe furent particulièrement actifs. Les éditeurs de la revue, les frères Alessandro et Pietro Verri, tentèrent en effet de rompre définitivement avec le pédantisme linguistique du passé à partir de positions explicitement rationalistes et éclairées ${ }^{10}$. Leurs propositions linguistiques s'inscrivaient dans le sens de la modernisation de la langue italienne et dans une dynamique " cosmopolite ». Toutefois, la tendance à souligner la dimension politique et nationale du problème linguistique 
s'accentuait, notamment dans la crainte que l'affaiblissement de la langue italienne ne conduise à la destruction du dernier rempart qui défendait encore l'identité nationale, compte tenu de la division politique de l'Italiie ${ }^{11}$. Il est important de noter que, au cours de ces années, sont apparus quelques-uns des motifs qui deviendront récurrents dans la rhétorique italienne nationaliste et romantique sur le lien inextricable entre langue et nation :

La langue est l'un des liens à la patrie les plus forts. Si les mots de nation et de patrie ne sont pas tout à fait vides de sens; s'il est important que chaque société civile conserve un caractère propre [...], alors il est certain que pour entretenir encore ce feu, nous ne devons pas cesser de rechercher chaque chemin, chaque moyen approprié auprès des savants et que tous ceux qui réunissent l'autorité et la connaissance se doivent de les mettre en pratique ${ }^{12}$.

6 Ce passage est tiré de l'une des œuvres les plus importantes du débat moderne sur la langue italienne : Dell'uso e dei pregi della lingua italiana publié en 1791 par Gian Francesco Galeani-Napione. L'intention de cet ouvrage est de répondre à la polémique née de la publication de l'œuvre de Melchiorre Cesarotti quelques années auparavant intitulée Saggio sopra la lingua italiana, de 1785, dans laquelle Cesarotti avait appelé à une modernisation de l'italien tout en s'ouvrant à l'influence de la langue français ${ }^{13}$. Ces deux œuvres ont marqué le début d'une ère nouvelle et plus mature dans le débat sur la langue italienne qui depuis le XIV siècle obsédait les savants italiens ${ }^{14}$. Si Galeani-Napione, s'accordait avec son " adversaire » sur le refus de fonder l'italien moderne sur le toscan, ce qui était l'espoir de la plupart des savants italiens depuis le Xvi siècle, il s'opposait en revanche à Cesarotti quant à l'imitation du français pour moderniser l'italien. En général, la polémique entre les deux intellectuels s'est concentrée sur les thèmes qui seraient au centre du débat intellectuel tout au long de l'ère napoléonienne et au-delà, à savoir le lien indéfectible entre la langue et la nation, le rôle des savants et des politiciens dans le développement de la langue, la place du toscan dans la définition de cette langue italienne, et surtout le poids du français en Italie. Il est significatif que l'œuvre de GaleaniNapione a été mis sous presse quelques années après la publication de Cesarotti: l'éclatement de la Révolution française a dû rendre nécessaire une réponse polémique à l'ouverture de l'italien au français.

7 Pendant le période du Triennio (1796-1799), après l'arrivée des Français et l'établissement de leur influence politique en Italie, la question s'est évidemment de nouveau posée de manière plus marquée. En effet, de nombreux intellectuels à cette époque acceptaient le message politique des Français, mais n'étaient pas d'accord sur son application en matière de langue. Certains patriotes pensaient que le français ne devait pas remplacer l'italien, mais plutôt servir de modèle pour son rajeunissement et sa " démocratisation ${ }^{15}$ ». La domination politique des Français porteurs de la Révolution a été l'occasion de refonder complètement l'italien des tribunaux et des académies dans un sens démocratique et populaire, à commencer par la lutte systématique contre l'analphabétisme, la modernisation radicale du vocabulaire politique et la régénération des qualités expressives du langage, tout cela dans le but de créer un véritable langage républicain, énergique, libre, franc et compréhensibles par tous les peuples ${ }^{16}$.

8 À partir de la fin de la Consulte de Lyon (1802), lorsque certains territoires italiens ont été annexés directement à la France, la discussion a profondément changé. Jusque-là, l'influence française avait été avant tout sensible dans les questions philosophiques et littéraires, mais l'intégration de certaines régions d'Italie à la France et la présence désormais physique des Français allaient influer sur le développement des questions liées 
à la langue italienne. Pendant cette nouvelle phase, chez les littérateurs italiens, il existait deux tendances, ou deux réactions, qui peuvent être résumées par deux ouvrages : Dell'uso della lingua francese de Carlo Denina publié en 1803 et Della necessità di scrivere nella propria lingua de Giovanni Rosini, publié en $1808^{17}$. Ce dernier, homme de lettres alors professeur d'éloquence à l'Université de Pise, avait souligné avec emphase dans son discours inaugural de l'année académique 1806 la nécessité d'écrire dans sa propre langue. À cette époque, Pise, qui se trouvait dans le Royaume d'Etrurie, n'avait pas encore été annexée à l'Empire (elle le sera en 1808), mais était environnée par les nouveaux départements annexés à la France et par le Royaume d'Italie.

Plus le génie des étrangers se répand chez nous, plus les défauts dont notre langue est chargée s'accroissent; plus l'empire irrésistible des armes la menace de près, plus les nobles âmes considéreront comme l'acte d'un esprit généreux et indépendant toute tentative faite pour s'y opposer, tout effort pour renverser les obstacles; puisque nous ne pouvons recueillir la palme des vainqueurs, ce sera une grande récompense encore, qu'une feuille de la couronne dont la patrie reconnaissante décorait autrefois la tombe des citoyens qui avaient combattu pour elle ${ }^{18}$.

9 Les armes menaçaient ainsi la langue italienne et les écrivains devaient vigoureusement s'opposer à ce danger ; la nécessité d'écrire dans sa propre langue, l'italien, était donc non seulement un devoir pour le monde des lettres, mais aussi et surtout pour le pays. Rosini a par conséquent exprimé une vive opposition à l'influence de la langue française sur la langue italienne, exacerbée depuis les conquêtes de Napoléon; il a également dénoncé le phénomène répandu amenant de nombreux italiens à écrire et publier leurs ouvrages en français.

10 Selon une démarche inverse à celle de Rosini, dans l'œuvre de Carlo Denina la réflexion répond aux directives prises après la récente réunion du Piémont à la France qui ordonnaient d'utiliser la langue française au lieu de l'italien pour les documents publics. Denina, historien piémontais qui sera nommé bibliothécaire personnel de Napoléon en 1804, a admis éprouver du regret devant cet abandon proposé de "notre langue », qui pourtant « est par son propre et natif génie capable de cette précision, de cette clarté et de cette facilité dont le français est si vivement doué et qui le favorise entre les autres langues anciennes et modernes». Mais il ajoute cependant que «ce changement de langue sera bien plus bénéfique que nuisible à la classe lettrée du Piémont » car " passées la perturbation et la peine dans les premiers temps, je pense avec certitude que nos petits-enfants écriront beaucoup plus facilement en français que nos ancêtres et contemporains ont pu le faire en italien ${ }^{19}$ ». Après tout, poursuit Denina, la langue réellement utilisée dans le Piémont était précisément le piémontais, qui n'était qu'un des dialectes de l'Italie, pas un italien ou un toscan corrompu, mais un latin corrompu par de diverses manières, et déjà semblable au français, surtout pour des raisons historiques ${ }^{20}$.

Ce document est suivi par les travaux d'un personnage important pour la vie littéraire italienne de l'époque. Je fais référence à l'abbé Aimé Guillon de Montléon, originaire de Lyon, qui deviendra plus tard superviseur de la section culturelle du Giornale Italiano (le journal officiel du gouvernement français à Milan). Guillon était un homme de lettres controversé, au point que Vincenzo Monti a pu écrire à son propos que «le Giornale Italiano est actuellement entre les mains d'une bête française, qui ne connaît pas une syllabe de bon italien, encore moins de latin, et décide de tout. L'énormité de ses bévues le met à l'abri de toute réfutation, parce que personne ne veut se dégrader avec un adversaire aussi misérable ${ }^{21} »$. Guillon a en fait mis en place une politique intense de 
francisation. En 1805, il a publié De quelques préventions des Italiens contre la langue et la littérature des Français : lettre à l'abbé Denina à l'occasion de son opuscule "Dell'uso della lingua francese nel Piemonte ${ }^{22}$. Se concentrant sur les principaux éléments avancés par Denina, il y souligne les points forts de la langue et de la littérature française, en exhortant les écrivains italiens à ne pas mépriser les Français. En se référant directement à Denina, Guillon déclare dans l'introduction que :

L'Italie, qui vous compte au nombre de ses premiers écrivains, s'enorgueillira de l'honneur que l'Auguste de notre âge lui fait en votre personne [allusion à la charge de Denina comme bibliothécaire personnel de Napoléon], quoique les Italiens d'à présent soient encore loin de penser comme vous en ce qui concerne les avantages de la langue française. C'est probablement à cette opposition d'opinion de leur part, qu'il faut attribuer l'espèce d'obscurité où votre ouvrage est resté comme enfoui dans le pays que j'habite ${ }^{23}$.

12 Comme observé par Guillon, la position de Denina est restée assez isolée, en dépit des nombreuses preuves de la force politique de la langue française en Europe face à la faible l'influence de l'italien qui restait essentiellement d'ordre littéraire. Le moment où Denina écrit représente le point culminant d'un consensus quant à la politique d'intégration linguistique et culturelle de la France : le Piémont venait d'être annexé par la République française qui, comme mentionné ci-dessus et comme cela sera analysé plus bas, a introduit l'usage de la langue française. Cette disposition intervient dans le contexte plus large d'une politique linguistique menée par le gouvernement de Napoléon en Italie, politique qui est d'une part destinée à la normalisation de l'appareil institutionnel et administratif, mais qui se propose aussi de diffuser la langue française dans toutes les sphères de la vie quotidienne.

\section{La politique linguistique de Napoléon Ier}

13 En matière de législation linguistique, les politiques impériales s'appuient principalement sur l'introduction obligatoire du français dans l'administration, dans les tribunaux et dans les documents privés établis devant notaire, et ce pour tous les départements italiens directement rattachés à la France ${ }^{24}$. Au moment du décret instituant cette obligation, promulgué le 24 prairial an XI (13 juin 1803), les territoires unis à la France comprenaient les départements correspondant au Piémont, à la Ligurie et à l'ancien Duché de Parme et de Piacenza. Toutefois, l'analyse de la législation ultérieure montre que d'importants délais ont été accordés de manière répétée pour retarder l'application de ce décret ${ }^{25}$. En effet, bien que le gouvernement français n'eût pas renoncé à son application définitive et aspirait à l'uniformité linguistique, il n'avait pas manqué de répondre, dans une perspective très pragmatique, aux besoins des villes qui ne parvenaient pas encore à produire de documents publics en français.

D'autres initiatives s'inscrivant dans le cadre des politiques linguistiques napoléoniennes sont liées à l'introduction de l'enseignement de la langue française, et parfois même à son usage, dans l'ensemble du système scolaire des départements annexés et du Royaume d'Italiie ${ }^{26}$. Généralement, les petites écoles, les collèges, les lycées et les facultés de lettres des différentes académies impériales, furent au départ conçus comme des lieux de propagation de l'enseignement de la langue ou de la littérature française. Toutefois, il convient de noter que ces transformations n'ont pas été appliquées de manière uniforme et que les résultats finaux ne furent pas à la hauteur des intentions initiales. En effet, les réformes introduites par les lois impériales ont modifié la structure des institutions et des 
programmes scolaires, mais elles se sont heurtées à des problèmes de nature technique et à l'opposition des élites locales ${ }^{27}$. Ces difficultés n'ont certainement pas facilité un changement qui devait s'étaler sur plusieurs années.

Le théâtre est également utilisé comme un moyen de propagation de la langue et de la culture française grâce à l'introduction de deux sociétés d'acteurs dirigées par l'actrice française Françoise Raucourt, dont la mise en scène et les pièces sont en français. Ces sociétés proposent les œuvres majeures du théâtre français dans les principales villes, pour l'une des départements annexés à l'époque (Turin, Alessandria, Gênes, Parme) pour l'autre du Royaume d'Italie (Milan, Brescia, Bologne, Venise) ${ }^{28}$. La volonté de diffuser la langue et la culture françaises dans les territoires italiens est explicitement à l'origine de la création de ces sociétés par un décret impérial du 10 juillet 1806, et est réaffirmée à plusieurs reprises par Mlle Raucourt et ses lieutenants ${ }^{29}$. Cependant, malgré les investissements continus du gouvernement napoléonien, l'entreprise du Raucourt fut infructueuse. À Milan, la troupe échoua si bien que le gouvernement du Royaume d'Italie décida de la retirer des mains de Mlle Raucourt et de la confier à un homme d'affaires local ${ }^{30}$.

16 Nous avons mentionné comment et pour quelles raisons les politiques de francisation adoptées par Napoléon se sont révélées peu efficaces; mais il faut également ajouter le facteur temps, au vu de la brièveté de la domination impériale. En effet, il semble que ces politiques auraient pu se révéler beaucoup plus efficaces si elles avaient duré. De plus, leur inefficacité est également imputable à l'inégale application des nombreuses initiatives napoléoniennes portant sur la langue qui, parfois, ont créé des exceptions ou des politiques différentes. Le cas de la Toscane est à cet égard paradigmatique : elle a tout d'abord été annexée directement à l'Empire et divisée en trois départements en application du décret du 24 mai $1808^{31}$. Par conséquent, «les lois qui régissent l'Empire français seront publiées dans les départements de l'Arno, de la Méditerranée et de l'Ombrone, avant le 1er janvier 1809, époque à laquelle commencera, pour ces départements, le régime constitutionnel». Le 18 mai 1808, la Giunta Straordinaria di Toscana a été créée avec notamment pour mission d'étendre les lois et règlements de la France aux trois nouveaux départements.

17 De sorte que la loi du 24 prairial an XI prescrivant l'usage de la langue française dans les actes officiels des départements annexés aurait également dû y être appliquée ${ }^{32}$. Cependant, moins d'un an après l'annexion, le 9 avril 1809, la Toscane s'est vu accorder par décret impérial le privilège, cas alors unique dans le système impérial, de pouvoir conserver l'usage de la langue italienne dans les domaines pourtant concernés par le décret du 24 prairial : «Art. I.er La langue italienne pourra être employée en Toscane, concurremment avec la langue française, dans les tribunaux, dans les actes passés devant notaires et dans les écritures privées ${ }^{33}$ ». Quelques mois plus tard, ce privilège fut étendu aux nouveaux départements romains. En effet, le 10 août 1809, à la suite de la formation et de l'annexion des départements des États de l'Église à l'Empire, la Consulta Straordinaria degli stati romani (une entité similaire à la Giunta toscane) avait établi que «la langue italienne peut être utilisée avec la langue française dans tous les actes administratifs et judiciaires, dans les actes passés devant notaire public et dans les écrits privés ${ }^{34} »$. Cette disposition concernait les deux départements créés dans les anciennes possessions de l'État pontifical, à savoir le département de Rome et le département du Trasimeno. La ressemblance avec le décret impérial du 9 avril 1809 était évidente, même si dans ce second cas, l'article 1 étendait les secteurs dans lesquelles la langue du pays pouvait être 
utilisée. C'est que le décret du 9 avril 1809 lui même avait en réalité été interprété dans un sens plus large que son seul sens littéral. La formule d'introduction du nouveau décret romain en témoignait, insistant sur « l'intention [de Napoléon] de conserver la langue italienne dans les départements d'Italie, récemment unis à son Empire», ce qui représentait un élargissement par rapport aux seuls actes publics.

Les exceptions des départements toscans et romains constituèrent ainsi une politique linguistique différente de celles appliquées dans d'autres parties de l'Italie et de l'Empire et elles eurent des conséquences à court et à long terme dans toute la péninsule italienne tant sur la perception de l'Empire napoléonien que sur le débat relatif à la langue italienne. Le décret sanctionnant le privilège toscan ne se référait pas seulement au domaine juridique, mais prévoyait également d'autres formes de protection de la langue. En effet, il introduisait parallèlement la distribution annuelle d'une récompense de 500 napoléons (soit 10000 francs), « décernée aux auteurs dont les ouvrages contribueront le plus efficacement à maintenir la langue italienne dans toute sa puretée ${ }^{35} »$. La gestion du prix fut confiée à l'Académie de la Crusca, réorganisée entre 1808 et 1811 notament à travers la promotion d'un nouveau vocabulaire ${ }^{36}$. À Rome, la concession de l'usage de la langue locale dans les actes officiels a également été suivie par l'institution d'un concours pour récompenser les œuvres jugées utiles au maintien de la pureté de la langue italienne. C'est l'Accademia dell'Arcadia, l'ancienne académie littéraire romaine fondée à la fin du XVII siècle et elle aussi réformée par le gouvernement français sur le modèle de celle de la Crusca de Florence, qui fut chargée de l'organiser ${ }^{37}$.

La concession de ces privilèges par le gouvernement napoléonien s'est faite en réponse à de nombreuses pressions et pour répondre aux préoccupations de l'élite culturelle italienne de l'époque. Elle fut pourtant controversée : pour certains, ces privilèges étaient interprétés comme une diversion de la part de Napoléon pour cacher ses véritables intentions impérialistes; pour d'autres, ils constituaient une initiative sincère visant à défendre le patrimoine linguistique italien. Cette dernière position fut soutenue par Carlo Botta, historien et homme politique italien, élu en 1802 au Corps législatif à Paris où il restera près de 10 ans $^{38}$. Il exprime son opinion dans ce qui semble être une réponse aux personnes doutant de la sincérité des politiques linguistiques de l'Empereur :

Est-ce ainsi que nous répondons à l'encouragement que l'Empereur a montré vers notre langue? Peut-être veut-il que nous parlions en esclaves? Qu'au lieu de revenir à la santé, nous nous enfoncions toujours davantage dans la corruption? Que nous parlions français avec des bouches italiennes? Que nous fassions refleurir de plus en plus le gout ampoulé du xvire siècle; ou les gallicismes, même les plus laids qu'on ait pu trouver au temps de la Révolution, et dont les Français se gardent aujourd'hui comme de la peste ? C'est pour cela peut-être que Sa Majesté a permis que même pour les actes publics, on employât en Toscane le toscan? C'est pour cela qu'Elle a fondé des prix ? C'est pour cela qu'Elle parle toujours italien à ceux qu'elle sait être Italiens ${ }^{39}$ ?

Face à ces décisions qui semblent contredire les indéniables efforts en vue de la francisation de la population italienne, il semble difficile de saisir les intentions de Napoléon. On pourrait certes les interpréter comme un moyen d'obtenir le consentement des nouveaux territoires annexés. Mais on pourrait aussi y trouver, au moins en partie, d'autres explications. La première porterait sur la possibilité que Napoléon ait voulu appliquer en Italie, avec l'italien, une initiative conceptualisée en France avec le français, mais non mise en pratique : la bataille contre les langues régionales et les patois, qui représentaient aux yeux des fonctionnaires impériaux l'un des bastions de l'Ancien 
Régime $^{40}$. En France, la volonté de normaliser la langue française sur le modèle parisien a souvent été exprimée ${ }^{41}$, mais cet projet ne s'est jamais traduit durant le Premier Empire par une politique linguistique spécifique. La seule initiative en ce sens est restée statistique, sous forme d'une enquête sur les dialectes menée entre 1807 et 1812 par Charles Coquebert de Montbret, directeur du bureau de la statistique de l'Empire, et son fils ${ }^{42}$. Une autre explication du privilège accordé par Napoléon à l'italien apparait plus tard, en février 1812, dans un rapport du ministre de la Justice, Claude Ambroise Régnier :

L'exception [...] de la Toscane, est un effet de la protection spéciale qu'elle accorde aux sciences et aux belles-lettres; elle a singulièrement pour objet de maintenir la langue italienne dans toute sa pureté : sous ce double rapport, elle serait de peu d'importance pour les provinces antérieurement réunies, qui ne se piquent point de la même pureté de langage, qui n'ont pas le même amour des lettres, et qui se livrent plus particulièrement à l'agriculture, à l'industrie et au commerce. Par des considérations purement politiques, on avait d'abord ordonné l'emploi exclusif de la langue française dans tous les actes publics sans distinction ${ }^{43}$.

Régnier poursuit en affirmant que « cette disposition a été déclarée commune aux États romains, par un arrêté de la Consulta Straordinaria, en date du 10 août 1809 ». Le ministre de la Justice reprenait d'abord les raisons invoquées dans le décret impérial concédant le privilège susmentionné à la Toscane et qui faisaient partie du préambule du décret : d'un côté la protection spéciale accordée aux trois départements de la Toscane, de l'autre le désir de maintenir la pureté de la langue italienne. Mais il ajoutait un élément clé à la discussion : il aurait été vain de concéder ce même privilège aux départements annexés avant la Toscane, car ils ne brillaient pas particulièrement dans le domaine des arts et lettres. Enfin le Grand-Juge reconnaissait que l'utilisation de la langue française avait été dictée par des considérations purement politiques, visant à standardiser et franciser l'ensemble de l'Empire.

Cependant, dans ce même rapport, Régnier rappelle qu'à l'instar de la Toscane et des États romains, d'autres régions de l'Empire furent elles aussi exemptées de l'application de la loi du 24 prairial. «Par plusieurs décrets rendus dans le cours des années 1810 et 1811, votre Majesté a daigné accorder la même facilité à ses sujets des départements de la Hollande, de l'Allemagne, du Simplon et à ceux des Provinces illyriennes ${ }^{44} »$. Ce qui avait été présenté comme un privilège unique pour la Toscane, au nom de la pureté de la langue qui y était parlée, puis étendue à Rome, pour la gloire de la deuxième capitale de l'Empire, avait en réalité aussi été accordé aux Pays-Bas, à l'Allemagne, au Simplon (Suisse) et aux provinces Illyriennes : de vastes territoires dans le système impérial ${ }^{45}$.

Ces concessions pouvaient peut-être s'expliquer par la difficulté à appliquer les objectifs d'uniformité et de rationalisation du projet administratif de Napoléon dans des contextes diversifiés et souvent difficiles, ce qui conduisit à des solutions davantage pragmatiques et conciliantes avec les autorités locales. Si les premières concessions revenant sur l'uniformité linguistique avaient été justifiées par une prédisposition particulière aux lettres et aux arts des habitants des départements toscans et romains, cette explication pouvait paraître difficilement valable pour les autres circonscriptions de l'Empire, précisément parce que le décret du 9 avril 1809 avait été promulgué en arguant du caractère unique de la situation italienne. Permettre l'utilisation des langues locales dans de si vastes territoires dut certainement apparaître comme un échec aux yeux de Napoléon et de ses fonctionnaires.

24 Il convient d'ajouter ici que les politiques adoptées dans les départements toscans et romains étaient « exceptionnelles » en ce qui concernait la langue des actes publics, mais 
également par d'autres aspects. Effectivement, en particulier dans les départements romains, les initiatives liées au système scolaire et au théâtre français ont été moins tangibles. Dans les départements toscans, à travers l'instruction publique, fut introduit, du moins sur le plan théorique, l'enseignement du français dans les écoles, les lycées et la faculté des lettres de l'Université de l'Académie de Pise, qui supervisait toutes les écoles des départements toscans ${ }^{46}$. Cependant, les problèmes liés à l'organisation et à l'ouverture de nouvelles écoles ralentirent la progression des études en général, et celle de la langue française en particulier ${ }^{47}$. De son côté, à Rome, la Consulta Straordinaria, au moment de son inauguration, estima qu'il y avait des questions plus urgentes à traiter que la réorganisation et la modernisation du système scolaire. Ce n'est qu'en 1812, peu avant la fin de la période napoléonienne, que fut nommé le recteur de la nouvelle Académie romaine, Giovanni Ferri de Saint-Constant. Il eu le temps de rédiger un « Rapport sur les organisations de l'instruction publique dans les départements de Rome et du Trasimène ", dans lequel, faisant part de ses réflexions sur les changements à apporter, il souhaitait lui aussi l'inclusion de l'enseignement du français dans les écoles des départements de Rome ${ }^{48}$.

Cependant, l'aspect le plus impressionnant est lié au théâtre français. En effet, dans les départements toscans et romains, il n'a pas été mis en place d'initiative similaire aux sociétés de Mlle Raucourt dans le nord de l'Italie. En Toscane, toutefois, la troupe d'acteurs français au service de la Grande-Duchesse Elisa Baciocchi a été amenée à jouer sur les scènes des villes visitées par la sœur de Napoléon, vraisemblablement dans le double but de divertir la cour du Grand-duché et d'habituer la population aux scènes récitées en français ${ }^{49}$. Mais à Rome, l'annexion des États pontificaux n'a jamais conduit à envisager l'introduction d'une compagnie d'acteurs français. L'analyse de la documentation archivistique a révélé de nombreuses tentatives du gouvernement pour fournir à Rome un ballet, un opéra et un opera buffa (vaudeville), mais pas de spectacles en français, alors que ceux-ci-ci étaient présent dans tous les départements impériaux et royaux, ainsi que dans le Royaume de Naples ${ }^{50}$.

\section{Langue et administration}

26 L'exemple des politiques des départements romains est essentiel pour comprendre que les aspirations idéologiques de l'Empire napoléonien se heurtaient à des problèmes liés à la gestion quotidienne des structures institutionnelles et administratives. Des compromis furent donc nécessaires, dont l'exemption de l'usage de la langue française dans les actes publics, mentionnée précédemment. Dans le domaine administratif, cette mesure n'avait cependant pas résolu définitivement les difficultés linguistiques car des problèmes de communication au sein des bureaux demeuraient. Tel était également le cas lorsque le décret du 24 prairial an XI entra définitivement en vigueur. En fait, bien que beaucoup de fonctionnaires français aient été employés dans les départements annexés d'Italie, de nombreux postes importants dans les bureaux des institutions restaient aux mains des Italiens. Ceux-ci devaient donc faire face à deux types de difficultés: non seulement utiliser une langue, le français, qui n'était pas leur, mais encore maîtriser un langage bureaucratique auquel ils n'étaient pas habitués ${ }^{51}$.

Un aspect jusqu'à présent peu étudié doit en effet être pris en considération : il porte sur l'usage de la langue française dans l'administration des divers départements italiens. Celui-ci était conditionné par la composition des bureaux qui elle-même variait souvent ${ }^{52}$. 
Il convient ici de s'intéresser à quelques témoignages inédits sur l'emploi respectif du français et de l'italien dans les départements annexés à l'Empire. L'analyse des correspondances des principales préfectures montre que la documentation circulant entre le centre et les départements concernés par le décret du 24 prairial an XI (ceux de Ligurie, du Piémont et de Parme), et entre les préfets de ces différents départements, est presque entièrement en français ${ }^{53}$. Cependant, la question de la communication entre les préfets et les administrateurs locaux se pose différemment car les maires des petites villes et villages, contrairement aux préfets, étaient italiens. Dans ce cas, un recours important à la traduction de l'italien vers le français était nécessaire car ces maires ne pouvaient généralement pas communiquer en français.

Un cas rare et inédit, concernant le département de Gênes, permet de saisir les stratégies adoptées en réponse au sein des préfectures, à travers la désignation d'un fonctionnaire engagé dans le seul but de s'occuper des traductions de la Préfecture. Un décret du Préfet de Gênes, Marie Just Antoine de La Tourette, daté 24 octobre 1806, pris en considération de «la diversité des langues et idiomes employées dans le département de Gênes [qui] rend indispensable de créer à la Préfecture une place de Secrétaire-Interprète » nomme le sieur « De Ferrari (Baptiste) sous-chef à la Préfecture de Gênes [...] secrétaire-interprète chargé de la traduction de pièces et actes présentés à l'administration ${ }^{54}$. Le préfet établit un service devenu indispensable au sein de la préfecture, compte tenu de la diversité des langues (français et italien) et des dialectes (génois et piémontais). Bien qu'il existe très peu de traces archivistiques d'autres cas de fonctions si clairement liées à la différence linguistique dans les bureaux napoléoniens, il est raisonnable de supposer qu'il y avait d'autres employés jouant le rôle de traducteurs-interprètes dans les préfectures.

Toutefois le cas présenté devient d'autant plus intéressant que, quelques mois après l'engagement de De Ferrari, le préfet de Gênes recevait le prospectus publicitaire d'une entreprise dont le succès était lié à la période précise de sa création. Un certain Giuseppe Crivelli, sur lequel nous n'avons pas d'informations biographiques pertinentes, écrivit en effet à La Tourette le 2 juillet 1807 pour lui présenter son "Établissement général d'agence et de correspondance en matière civile, administrative et judiciaire, fondé à Gênes, chef-lieu de la $28^{\mathrm{e}}$ Division militaire par Crivelli ${ }^{55}$ ». À la lettre était joint un dépliant bilingue, dans lequel Crivelli exposait son curriculum, décrivait son agence et se présentait comme l'auteur d'une Raccolta Ragionata, une œuvre où il avait analysé l'administration française $\mathrm{e}^{56}$. Le dépliant était suivi d'un prospectus en français dans lequel Crivelli réaffirmait ses compétences: "les traductions de la langue italienne en langue française et viceversa pourraient lui fournir des moyens très amples pour alimenter par intérim son bureau, mais cette partie étant réglée et organisée par un décret de M.r le Préfet, toute espérance à cet égard serait chimérique. Le traducteur juré de la Préfecture remplit déjà cette tâche qui est assez conséquente ». Crivelli était donc conscient que De Ferrari exerçait déjà les fonctions pour lesquelles il se présentait au préfet, mais il ajoutait : «L'occupation que le soussigné prend la liberté de demander à M.r le Préfet La Tourette dans sa lettre, sera très analogue à sa position actuelle et aux études administratives envers lesquelles le soussigné paraît entrainé par un penchant irrésistible et par un goût tout à fait naturel ». Nous ne savons pas si le Préfet de Gênes eut recours aux services de Crivelli, mais de toute manière, son cas reste significatif : il a saisi l'esprit et les besoins de l'époque, c'est-à-dire les traductions et la gestion de la correspondance des particuliers, mais surtout celle des institutions publiques, nécessités qui entravaient leur fonctionnement quotidien. En effet, les fonctionnaires devaient compter avec des 
pratiques alourdies par la problématique de la langue. Mais ce n'est qu'à des niveaux élevés, par exemple dans les préfectures, que le budget permettait d'engager un employé qui pouvait répondre à ces besoins, comme De Ferrari.

Dans les cas romain et toscan, la question de la langue en usage dans les bureaux est plus complexe car malgré la suspension de l'obligation d'utiliser le français, la communication ordinaire se réalisait dans les deux langues, donnant lieu à des situations exceptionnelles. L'une des plus intéressantes est celle des habitudes linguistiques du préfet de la Méditerranée (Livourne), le Français Guillaume Capelle, qui n'écrivait pas toujours dans sa langue maternelle. Pendant les premiers mois de l'annexion de la Toscane à l'Empire, Capelle n'a pas manqué d'écrire en italien à ses interlocuteurs, de toute évidence pour se faire connaitre des notables locaux et pour donner une image inclusive de la collaboration $^{57}$. Mais même après cette phase initiale, Capelle n'homogénéisa pas sa pratique linguistique : il lui arrivait d'écrire au même destinataire un jour en italien et le lendemain en français ${ }^{58}$.

31 Évidemment, dans son travail quotidien, le préfet n'écrivait pas lui-même ses lettres en italien, ayant recours à un fonctionnaire, souvent le secrétaire général. Capelle maintint néanmoins l'alternance entre les lettres en français et en italien pendant toute la durée de son mandat, jusqu'à son transfert à la fin de 1810, à Genève comme préfet du département du Léman. Son successeur, Michel-Augustin de Goyon, resta préfet de la Méditerranée jusqu'à la chute de Napoléon. La comparaison des correspondances se révèle parlante. En effet, l'arrivée du nouveau préfet marque un tournant dans la documentation: Goyon n'écrit ses lettres qu'en français ${ }^{59}$. Ce changement de politique n'est pas anodin. Une explication possible pourrait être la position idéologique du nouveau préfet, issu d'une famille noble, en faveur de politiques linguistiques moins conciliantes. D'ailleurs, si la loi du 9 avril 1809 autorisait l'usage de l'italien dans les actes officiels, elle n'obligeait cependant pas les fonctionnaires français à l'utiliser ${ }^{60}$.

Même lorsque l'italien demeurait la langue officielle, comme dans le Royaume d'Italie, l'analyse des documents du gouvernement central de Milan révèle une situation complexe. En effet, toutes les lois du Royaume furent promulguées en italien, à l'exception de trois statuts constitutionnels ${ }^{61}$. Toutefois, la documentation ordinaire contient beaucoup de documents en français car le vice-roi, Eugène de Beauharnais, écrivait dans sa propre langue. Cette pratique pouvait sembler "naturelle » compte tenu de ses origines, mais elle aurait pu également avoir une explication plus profonde, illustrée par les paroles de Napoléon qui, dans ses instructions à Eugène lors de son installation comme vice-roi, écrivait: «La connaissance qui vous manque de la langue italienne est un très bon prétexte pour vous abstenir ${ }^{62}$ ». Il n'y avait pas seulement la correspondance et la documentation du vice-roi qui étaient principalement rédigées en français, mais aussi celles des nombreux interlocuteurs français des fonctionnaires lombards employés dans le Royaume. Bien que peu nombreux, il existait aussi des Français engagés dans le gouvernement du royaume italien, entre autres Etienne Méjan, secrétaire du vice-roi, et Marie-François Auguste de Caffarelli, ministre de la Guerre et de la Marine du Royaume d'Italie, certes d'origine italienne ${ }^{63}$. La politique linguistique du Royaume d'Italie ne se résume donc pas au maintien de l'italien comme langue officielle ${ }^{64}$. La situation était plus complexe car le vice-roi souhaitait contrôler et orienter le consensus tout en apparaissant attentif aux enjeux locaux. De sorte qu'au quotidien, de nombreux fonctionnaires du Royaume échangaient souvent en français. Par conséquent, même si le français n'était pas prescrit, les fonctionnaires au service du vice-roi devaient 
le connaître pour s'acquitter de leurs fonctions administratives et gouvernementales. Or, il ne faut pas oublier que sur de nombreuses questions importantes, la référence au gouvernement de Paris était constante.

Outre la connaissance du français, le système napoléonien supposait une façon de travailler et de communiquer complètement nouvelle. Le problème de la langue n'était pas isolé puisqu'il fallait également connaitre les lois et procédures du système français : «il faut aussi que, dans le même espace de temps, ils [les fonctionnaires italiens] apprennent par cœur le Code pénal, le Code civil, le Code de procédure, le Code des douanes, des domaines, des droits réunis, avec tous les arrêtés des cours impériales et de la cour de cassation, sans oublier les circulaires et décisions ministérielles $»^{65}$. En effet, dans les départements réunis comme dans le Royaume d'Italie, outre l'application des codes législatifs français, la législation impériale était une référence constante. Le langage changeait très vite à travers le nouvel idiome juridique et bureaucratique que les Français avait introduit en Italie en même temps que la nouvelle administration. Celle-ci engendra un ensemble d'écrits (rapports, correspondances, bulletins, etc.) qui constituèrent une véritable rupture en termes de contenu et de quantité. L'augmentation du niveau de production de documents divers et de correspondance distingue fortement ce début du $\mathrm{XIX}^{\mathrm{e}}$ siècle, quand la langue italienne commença à prendre sa forme définitive.

On peut à bon droit supposer que la masse des écrits français, ou basés sur le modèle français, a joué un rôle notable dans l'émergence de l'idée de ce qu'aurait dû être l'italien moderne dans l'esprit des fonctionnaires italiens. De fait, ceux-ci remplissaient souvent un double rôle d'employé ou de bureaucrate du gouvernement français en Italie, mais aussi d'écrivain. Un exemple important en ce sens est celui de Giuseppe Bernardoni qui était chef de division du ministère de l'Intérieur du Royaume d'Italie. Bernardoni publia à Milan en 1812 un ouvrage intitulé Elenco di alcune parole oggidì frequentemente in uso le quali non sono ne' vocabolari italiani ${ }^{66}$, où la plupart des mots cités font partie de la «langue utilisée par les secrétaires ». Avec cette formule, Bernardoni renvoie à la nouvelle langue bureaucratique introduite par les Français. Le travail de Bernardoni, comme on le montrera plus loin, a eu des répercussions sur le débat qui suivit l'occupation napoléonienne ; mais il est aussi révélateur de l'impact des pratiques administratives sur la communication dans la vie quotidienne, source de nouvelles réflexions sur la langue italienne.

\section{La réaction des hommes de lettres}

Cesare Balbo est une autre figure importante assumant le double rôle de fonctionnaire napoléonien et d'homme de lettres. Historien et homme politique italien de premier plan, Balbo fut très jeune secrétaire général de la Giunta Straordinaria di Toscana en 1808, puis de la Consulta Straordinaria degli stati romani en 1809 et $1810^{67}$. Il ressort clairement de la documentation officielle que Balbo, en tant que fonctionnaire napoléonien, respectaient les devoirs de sa fonction également au niveau linguistique, puisqu'il décida d'adopter une politique cohérente en adressant des lettres en français à tous ses interlocuteurs, qu'ils soient français ou italiens. Comme il écrivait au nom du gouvernement impérial, son choix peut être compris comme dicté par le désir de se conformer aux pratiques de ses collègues français, mais aussi comme une marque de respect vis-à-vis du gouvernement français et de sa langue. 
36 Au moment où Balbo était occupé par les derniers travaux de la Consulta à Rome, et avant de s'installer à Paris en 1811 après avoir été nommé conseiller d'État à seulement 22 ans, il s'impliqua aussi dans un échange de lettres avec son ami, Carlo Vidua ${ }^{68}$. Parmi les différents thèmes abordés entre eux, on trouve de manière récurrente celui de la langue italienne. Leur débat est important pour de nombreuses raisons, à commencer par le travail de Balbo au sein du gouvernement napoléonien. D'autre part, leurs échanges, notamment les réflexions de Vidua, montrent que les enjeux linguistiques préoccupaient tous ceux qui s'occupaient de près ou de loin de belles lettres. Enfin, le sujet est traité de manière approfondie, en particulier dans la relation entre les langues française et italienne. Dans une lettre datée du 12 juillet 1810, Vidua s'exprime sur la possibilité envisagée par Balbo d'écrire une œuvre historique. Ce dernier lui a probablement demandé conseil sur la langue à utiliser pour l'écriture, en évoquant la possibilité d'abandonner sa propre langue au profit du français. Vidua lui répondit :

Il reste à choisir la langue. Mais que dois-je vous dire que vous ne compreniez déjà? Vous direz que pour votre carrière vous avez besoin de bien étudier le français? Telle est la vérité, que non seulement vous comprenez, mais qui vous pousse presque à abandonner la plus belle langue, et qui est la vôtre, pour elle. Ceci est d'ailleurs une pensée qui me fait frémir [...] et je préférerais vous connaître trop mal pour pouvoir penser à bon droit qu'après tant d'amour et de zèle pour notre langue, vous pourriez finir par l'abandonner pour une langue étrangère, et quelle langue $^{69}$ !

Ces lignes reflètent le mécontentement de Vidua vis à vis de l'emploi du français, mais aussi le constat que Balbo, en tant qu'officier napoléonien, se devait de le maîtriser. La proposition de Carlo Denina ne semble donc finalement pas si éloignée de la réalité de l'époque : les littérateurs, dans leur confrontation quotidienne avec les Français et du fait qu'ils occupaient souvent un poste dans l'administration française, comme Balbo, ont pu croire qu'il était plus commode d'écrire en français, en partie aussi parce que cette option impliquait une meilleure diffusion de leur travail. En outre, pour un Piémontais, le choix entre les différentes variantes de l'italien était loin d'être évident, étant donné que la langue italienne n'avait pas encore trouvé sa forme unifiée. Même Balbo ressentait l'inquiétude qui planait à l'époque dans son cercle littéraire, et ce sentiment transparait dans son introduction à l'édition posthume des lettres de Carlo Vidua :

La quasi-totalité d'entre nous écrivaient francisé («infranciosato»), et les autres, pour échapper à ce vice, s'étaient jetés dans les archaïsmes et les florentinismes («fiorentinismi affettati »). Chacun sait que cette alternative a perduré longtemps, et ils sont peu, très peu, ceux qui s'en sont sortis sans l'un ou l'autre vice; certains ont même trouvé le moyen de les réunir tous les deux. Carlo [Vidua] nous a tous précédé, et son époque avec, et il a écrit, dès ce moment-là, dans le vrai italien ${ }^{70}$.

Cette recherche du "vrai italien» dont parle Balbo avait torturé Vidua lorsqu'il s'était attelé à la rédaction de son seul ouvrage datant du milieu de l'époque napoléonienne, à savoir Dello stato delle cognizioni in Italia, qui portait sur divers sujets, y compris la question de la langue italienne $e^{71}$. Après une première relecture, Vidua écrivit à Balbo qu'il s'était retrouvé dans « une mer d'ennuis, à découvrir chaque jour de nouvelles inélégances, un grand nombre d'erreurs, de nouveaux doutes sur la langue, et toujours des mots francisés qui passaient inaperçus ». Les travaux de Vidua, et de tous les écrivains italiens, ont été influencés par la peur « d'insérer nouvelles bévues, ou de laisser quelques Gallicismes ou grosses erreurs ${ }^{72}$ ». Pour essayer d'éviter ces erreurs, Vidua a cherché du réconfort dans les manuels du temps et s'est « entouré [des Vocabulaires] de Corticelli et de l'Académie de la Crusca, des Synonymes de Rabbi, de trois ou quatre grammaires et de l'Orthographe du 
Facciolati ${ }^{73}$ » : ces multiples " autorités » linguistiques ne l'ont cependant pas beaucoup aidé à clarifier les choses, parce qu'elles étaient souvent en désaccord entre elles.

Finalement, à partir de tous les cas cités, qui ne représentent qu'une partie de ceux découverts, nous pouvons conclure que la présence du français a engendré, plus que jamais auparavant, des difficultés pour les hommes de culture. Nous ne pouvons guère imaginer la confusion qui régnait alors dans l'esprit des savants vis-à-vis de la langue italienne : ils devaient faire à la fois avec les usages complexes d'un langage non encore commun et avec la volonté de doter la nation italienne d'une langue moderne. À ces enjeux se surimposèrent les questions liées aux politiques de francisation de la société italienne mises en œuvre par Napoléon, qui de leur côté contrastaient avec la promotion de l'italien à travers la création des prix littéraires de la Crusca et de l'Arcadia.

À cet égard, le premier prix de la première édition de l'Accademia della Crusca fut remporté en 1809 par un savant dont les travaux eurent des retombées tout au long du xIX siècle. Il s'agit de l'abbé Antonio Cesari, vainqueur avec son ouvrage Dissertazione sopra lo stato presente della lingua italiana ${ }^{74}$. À travers une discussion sur l'état de l'italien, Cesari mettait en valeur les moyens de conduire la langue italienne vers une pureté de forme qu'elle aurait possédé à ses origines, dans la Toscane du XIV siècle. Antonio Cesari est ainsi considéré comme l'un des fondateurs du courant du Purismo ${ }^{75}$ qui allait marquer le climat littéraire et les programmes scolaires de l'Italie risorgimentale ${ }^{76}$, en promouvant l'argument déjà séculaire selon lequel le toscan archaïque constituait la véritable langue italienne.

Bien que Cesari ait publié dès 1810 , le travail considéré rétrospectivement comme le premier spécifiquement puriste est plutôt l'Elenco de Giuseppe Bernardoni qui date de $1812^{77}$. En effet, l'intention de Bernardoni n'était pas seulement de mettre à jour le vocabulaire de l'époque: son Elenco était conçu comme polémique, affirmant publiquement le rejet de certains mots utilisés dans la société italienne. Il convient d'ajouter que Bernardoni l'avait rédigé à la demande du ministre de l'Intérieur du Royaume d'Italie, Vaccari, qui s'alignait ainsi sur les politiques napoléoniennes de promotion de la langue italienne la plus pure.

Les initiatives du gouvernement impérial et royal ont donc favorisé un mouvement qui a perduré. On ne saurait en effet négliger l'importance du lien entre la promotion de la pureté de la langue par les prix napoléoniens et le mouvement intellectuel qui favorisa cette même pureté tout au long du xIX siècle, marquant le développement de la nation et de l'État italien. La persistance de ce mouvement au cours du temps est également significative, alors même que le Purismo avait été combattu par les principaux protagonistes de la scène littéraire italienne de l'époque comme Vincenzo Monti et surtout Alessandro Manzoni. Ce dernier entretenait une abondante correspondance avec Cesari, mais il n'était pas d'accord avec son argument « archaïque » comme le soulignera un écrit inédit publié seulement après la mort de Manzoni ${ }^{78}$.

L'importance de Manzoni dans le débat sur la langue italienne rejoint aussi notre discussion d'une autre manière : il a en effet été fortement influencé par la culture et la langue française. Des événements biographiques l'ont amené à résider à Paris de 1805 à 1810, et plus généralement, sa formation littéraire et personnelle s'est déroulée au beau milieu de l'époque napoléonienne. Dès le début de sa carrière, Manzoni a exprimé, comme ses contemporains, sa consternation à l'égard de l'italien et son admiration pour la langue 
française, comme il s'en explique dans une longue lettre à Claude Fauriel datée du 3 novembre 1821, quelques années après la chute de Napoléon :

Ce fait est (je regarde pour m'assurer que personne n'écoute) ce triste fait est, à mon avis, la pauvreté de la langue italienne. Lorsqu'un Français cherche à rendre ces idées de son mieux, voyez quelle abondance et quelle variété de modi il trouve dans cette langue qu'il a toujours parlé, dans cette langue qui se fait depuis si longtemps et tous les jours dans tant de livres, dans tant de conversations, dans tant de débats de tous les genres. [...] Imaginez-vous au lieu de cela un Italien qui écrit, s'il n'est pas Toscan, dans une langue qu'il n'a presque jamais parlé, et qui (si même il est né dans le pays privilégié) écrit dans une langue qui est parlée par un petit nombre d'habitants de l'Italie, une langue dans laquelle on ne discute pas verbalement de grandes questions, une langue dans laquelle les ouvrages relatifs aux sciences morales sont très rares, et à distance, une langue qui (si l'on en croit ceux qui en parlent davantage) a été corrompue et défigurée justement par les écrivains qui ont traité les matières les plus importantes dans les derniers temps [... ]. Qu'il se demande si la phrase qu'il vient d'écrire est italienne ; comment pourraitil faire une réponse assurée à une question qui n'est pas précise ? Car, que signifie italien dans ce sens? Selon quelques-uns ce qui est consigné dans la Crusca, selon quelques autres ce qui est compris dans toute l'Italie, ou par les classes cultivées : la plupart n'applique à ce mot aucune idée déterminée. Je vous exprime ici d'une manière bien vague et bien incomplète un sentiment réel et pénible ${ }^{79}$.

Le doute permanent des écrivains italiens les auraient conduit à opérer des choix drastiques, comme ceux des puristes que Manzoni ne blâme d'ailleurs pas. Il croit au contraire en la « rigueur farouche et pédantesque de nos puristes qui, à mon avis, produit un sentiment général fort raisonnable ; c'est le besoin d'une certaine fixité, d'une langue convenue entre ceux qui écrivent et ceux qui lisent [...] ». Manzoni explique à Fauriel ces mêmes doutes qui depuis des décennies taraudent les esprits des littérateurs italiens : " Dites-moi à présent ce que doit faire un Italien, qui ne sachant faire autre chose, veut écrire [...], dans le désespoir de trouver une règle constante et spéciale pour bien faire ce métier ». Une aide providentielle pourrait venir des «écrivains d'autres langues, les Français surtout ».

Cette déclaration de Manzoni démontre qu'après la chute de l'empire napoléonien en Italie les réflexions autour de la langue se distinguaient encore incontestablement par des comparaisons constantes avec le français. En effet, si, après la chute de l'Empire, la culture française en Italie a été vaincue, comme l'affirmait Hazard, nous avons vu à quel point le gouvernement napoléonien était intervenu dans les questions linguistiques italiennes, avec des conséquences durables. Même s'il est très difficile de déterminer si la diffusion du français et la référence constante des écrivains à cette langue sont imputables à la diffusion de la culture française du XVIII ${ }^{e}$ siècle ou bien aux résultats des politiques et de la présence impériale, il reste possible de proposer quelques réflexions conclusives.

On a en effet essayé de démontrer comment, dans l'Italie napoléonienne, la dimension culturelle est entrelacée avec la dimension politique par l'intermédiaire de l'élite culturelle italienne ${ }^{80}$. En effet, à travers ses fonctions gouvernementales ou ses engagements personnels, elle a été impliquée dans les questions linguistiques soulevées par les initiatives napoléoniennes. Les intellectuels italiens ont tissé une continuité entre la période précédant et celle suivant l'occupation napoléonienne, permettant ainsi l'absorption des apports français, puis leur dépassement, selon l'interprétation de Hazard. Cet article a ensuite mis en évidence les conséquences des propositions de 
Hazard, résumées ci-dessous, conséquences qu'il avait évoquées mais sans les articuler à sa thèse initiale.

La première, et la plus importante, réside dans les interactions constantes avec la langue française. En fait, la confrontation quotidienne avec le français des hommes de lettres italiens dans l'exercice de fonctions administratives de l'empire, même pour ceux œuvrant dans les territoires ayant conservé l'usage officiel de la langue italienne, ne faisait que souligner à quel point cette dernière restait floue, archaïque et complexe par rapport au français, clair et simple. Il apparaissait alors urgent de moderniser l'italien, ce qui aurait favorisé l'amélioration du fonctionnement des institutions gouvernementales et facilité la communication, mais également encouragé une nouvelle littérature. La deuxième conséquence est que, par les entorses à l'uniformité francophone et la promotion d'une langue pure et toscane, Napoléon avait renforcé un mouvement déjà très puissant, qui se concrétisera par la suite dans le Purisme. Nous l'avons déjà vu cidessus: bien que ce mouvement ait rencontré de nombreux et illustres adversaires comme Alessandro Manzoni (mais aussi Vincenzo Monti qui, dans sa Proposta, cherchait à s'opposer au dogmatisme de la $\left.\mathrm{Crusca}^{81}\right)$, il contribua tout au long du XIX ${ }^{\mathrm{e}}$ siècle à rendre la langue italienne plus forte, quoique pas plus moderne.

Les initiatives de Napoléon, qui, pour certains, n'étaient qu'un prétexte pour séduire l'élite culturelle italienne, ont en réalité favorisé l'ouverture d'un débat qui rejouait en partie sur d'anciens thèmes, lesquels y trouvèrent par la suite de nouveaux adeptes. Ces derniers se firent les partisans d'une volonté de doter la nation italienne de sa propre langue. C'est d'ailleurs à la fin de la domination napoléonienne que le lien entre langue et nation commença à alimenter le débat sur le Risorgimento ${ }^{82}$, avec le désir de libérer la nation italienne de sa dépendance envers les peuples et les langues étrangers.

\section{NOTES}

1. Paul HAZARD, La Révolution française et les Lettres italiennes (1789-1815), Paris, Hachette, 1910.

2. Giuseppe RICUPERATI, « Paul Hazard », Belfagor, vol. 23, n. 5, 1968, p. 564-595 (576).

3. Sur la réception de Hazard en Italie cf. Comparatistica. Annuario italiano, vol. 7, 1995, p. 182-183.

4. Giovanni GeNTILE dans La Critica. Rivista di Letteratura, Storia e Filosofia diretta da B. Croce, n. 9, 1911, p. 454-456.

5. Sur Giovanni Gentile et Benedetto Croce comme interprètes de la parabole napoléonienne cf. l'introduction de Antonino DE FRANCESCO, L'Italia di Bonaparte: politica, statualità e nazione nella penisola tra due rivoluzioni, 1796-1821, Turin, Utet, 2011.

6. Paul HAZARD, La rivoluzione francese e le lettere italiane (1789-1815), Roma, Bulzoni, 1995.

7. Au cours des dernières décennies, ce thème a été au centre des études de nombreux historiens, notamment anglo-saxons, qui ont remis en question les racines idéologiques des conquêtes napoléoniennes en Europe cf. Stuart wooLF, Napoleon's Integration of Europe, London/New York, Routledge, 1991. Cependant, c'est l'historien Michael Broers qui croit qu'en Italie l'impérialisme culturel napoléonien a eu le point de développement maximal, thèse qui se développe dans une de ses œuvres les plus importantes : Michael BROERS, The 
Napoleonic Empire In Italy. Cultural Imperialism In A European Context?, London, Palgrave Macmillan, 2005. Le travail de Broers a fait l'objet de nombreuses discussions (cf. Steven ENGLUND, "Monstre Sacré: The Question of Cultural Imperialism and the Napoleonic Empire», The Historical Journal, 51, n.1, 2008, p. 215-250), surtout à cause de sa thèse la plus controversée, à savoir la dépendance directe du mouvement de résurgence nationaliste italien vis-à-vis de l'impérialisme culturel napoléonien.

8. Sur la Questione della lingua cf. Maurizio VITALE, La questione della lingua, Palermo, Palumbo Editore, 1978. L'influence du français sur l'italien cf. Silvia MORGANA «L'influsso francese », Storia della lingua italiana. Vol. III. Le altre lingue, Torino, Einaudi, 1994, p. 671-719.

9. Cesare BECCARIA, "Frammento sullo stile ", Il Caffé, I, xxV, 1764, cité dans Gianni Francioni, Sergio Romagnoli (dir.), Il Caffe 1764-1776, Torino, Bollati Boringhieri, p. 277.

10. Cf. Maurizio VITALE, Proposizioni teoriche e indicazioni pratiche nelle discussioni linguistiche del Settecento, dans Lia FoRMIGARI, (ed. ), Teorie e pratiche linguistiche nell'Italia del Settecento, Bologna, Il Mulino, 1984.

11. Cf. Vittorio CRISCUOLo, « Per uno studio della dimensione politica della questione della lingua : Settecento e giacobinismo italiano », Critica Storica, XIV (1977), p. 410-470; XV (1978), p. 109-171; XV (1978), p. 217-344.

12. Gian-Francesco Galeani-NAPIONE, Dell'uso e dei pregi della lingua italiana, Volume I, Turin, Barbino e Prato, 1791, p. 3.

13. Melchiorre CESAROTTI, Saggio sopra la lingua italiana, publié sous sa forme définitive en 1802 sous le titre Saggio sulla filosofia delle lingue applicato alla lingua italiana, Padoue, Brandolese, 1802.

14. Cf. Vittorio CRISCUOLO, Il valore nazionale della lingua nella polemica fra Cesarotti e Galeani-Napione, dans Beatrice Alfonzetti et Marina Formica (éd.), L'idea di nazione nel Settecento, Rome, Edizioni di storia e letteratura, 2013, p. 63-64.

15. Par exemple Matteo Galdi, Giuseppe Compagnoni et Girolamo Bocalosi, cf. Vittorio criscuolo, «Per uno studio...op. cit.», XV, p. 109-171.

16. Au sujet de la langue du Triennio dans le discours politique de l'ère républicaine en Italie cf. Erasmo LESO, Lingua e rivoluzione: ricerche sul vocabolario politico italiano del triennio rivoluzionario, 1796-1799, Venise, Istituto veneto di scienze, lettere e arti, 1991.

17. Carlo DENINA, Dell'uso della lingua francese. Discorso in forma di lettera diretto ad un letterato piemontese, Berlin, Quien, 1803; Giovanni RosinI, Della necessità di scrivere nella propria lingua. Orazione premiale pronunciata alle pubbliche lezioni di eloquenza italiana all'Università di Pisa nell'anno 1806, Florence, Molini e Landi, 1808.

18. Traduit par Paul HAZARD, op. cit., p. 322.

19. Carlo DENINA, op. cit., p. 3.

20. Tout d'abord, l'origine ancienne et commune des peuples Gaulois cisalpins et transalpins à l'époque romaine, puis les nombreuses campagnes de conquête menées par les Français au cours des siècles.

21. Vincenzo Monti, 11 mars 1807, traduit par Paul HAZARD, op. cit., p. 271.

22. Aimé GUILLON DE MONTLEON, De quelques préventions des Italiens contre la langue et la littérature des Français : lettre à l'abbé Denina à l'occasion de son opuscule: Dell'uso della lingua francese nel Piemonte, Milan, Destefanis, 1805.

23. Ibid., p. 2.

24. Piero FIORELLI, "L'italiano, il francese, la Toscana e Napoleone », dans Studi in onore di Manlio Udina, II, Milan, Giuffrè, 1975, p. 1577-1602.

25. Ibid., p. 1579. 
26. Pour un cadre de la francisation de l'éducation en Italie cf. Elena BRAMBILLA, Carlo CAPRA, Aurora sсотті (dir.), Istituzioni e cultura nell'Italia napoleonica, Milano, Franco Angeli, 2008, JacquesOlivier Boudon (dir.), Napoléon et les lycées: enseignement et société en Europe au début du XIX siècle, Paris, Nouveau monde, 2004, p. 123 ; Sandro BUCCI, La scuola italiana nell'età napoleonica. Il sistema educativo e scolastico francese nel Regno d'Italia, Rome, 1976; Angelo BIANCHI, "Scuola e società nell'Italia napoleonica. Giovanni Scopoli e l'istruzione femminile (1809-1816) ", dans Le carte e gli uomini. Storia della cultura e delle istituzioni (secoli XVII-XX). Studi in onore di Nicola Raponi, Milan, Vita \& Pensiero, 2004, p. 125-151.

27. Intéressant à cet égard est le cas du lycée de Parme dans le département du Taro où l'élite locale s'oppose à l'ouverture du lycée cf. Michael BROERS, Le lycée de Parme sous le Premier Empire: une manifestation d'impérialisme culturel ?, dans Jacques-Olivier BOUDON (dir.), op. cit., p. 147-164.

28. Cf. Raul MARKOVITS, Civiliser l'Europe: Politiques du théâtre français au XVIII siècle, Paris, Fayard, 2014; Philippe BOURDIN, «Les limites d'un impérialisme culturel, le théâtre français dans l'Europe de Napoléon ", Le movement social, 2015/4 (n. 253), p. 89-112; Alberto BENTOGLIO, «Mlle Raucourt e la compagnia imperiale e reale dei commedianti francesi in Milano (1806- 1814)", ACME, n. 43, I, 1990 ; Henry LYONNET, " Mademoiselle Raucourt directrice des théâtres français en Italie (1806-1807) ", Bulletin de la société du théâtre, 1902, 1, p. 43-78.

29. Raucourt expliqua à Napoléon que le but de l'entreprise était de «faciliter les relations entre les deux peuples [et] propager la langue française »: Alberto BENTOGLIO, op. cit., p. 17. Un autre exemple est celui du régisseur de Raucourt, un certain Lejey, qui demande au préfet du département du Reno, au Royaume d'Italie, un certificat dans lequel le préfet déclarerait que la société a répondu à : «l'utilité des théâtres français dans ce Royaume, pour la propagation d'une langue qui a toujours été recherchée, principalement dans cette ville savante » : ASBo, Prefettura del Dipartimento del Reno, Titolo xxVI (Spettacoli), Anno 1809, lettre du 31 octobre 1809.

30. Il s'agit de Benedetto Ricci qui, dans une lettre adressée au gouvernement de Milan à la suite de sa nomination, tient ces propos frappants : «le but du gouvernement [en introduisant un spectacle de langue française en Italie] est plus sublime, plus vaste et plus généralement avantageux. Ses intentions, en admettant chez-lui un théâtre étranger dans une sorte de concurrence avec le théatre national, sont, non pas simplement d'augmenter les plaisir du public, mais encore de faciliter au théâtre national les moyens de s'approprier ce qu'il peut trouver de bon dans l'autre; c'est d'exciter en lui une émulations propice aux progrès de l'art dramatique en Italie, comme encore de familiariser les indigènes avec la langue des français qui se trouvent, comme eux, les sujets du même monarque, et pour ainsi dire les enfant du même père» : Archivio di Stato di Milano (ASM), Atti di governo, Spettacoli p.m., n. 26, 26 décembre 1813.

31. Bulletin des lois de l'Empire français, Paris, De l'imprimerie impérial, IV série, tome 8, p. 321.

32. Bulletin de la République français, op. cit., série III, tome 8, p. 598.

33. Bulletin des lois de l'Empire français, op. cit., IV série, tome 11, n. 4303, p. 147.

34. Bollettino delle leggi e decreti imperiali pubblicati dalla consulta straordinaria negli stati romani, Rome, Vol. III, 1809, p. 816-817 (en français dans le texte).

35. Fabrizio FRANCESCHINI, «I nipotini di padre Cesari. Il purismo e la sua influenza nella scuola dell'Italia Unita », dans Silvia morgana, Nicoletta maraschio, Annalisa Nesi (dir.), Storia della lingua italiana e storia dell'Italia Unita, Firenze, Cesati, 2011, p. 295-310.

36. Ibid. p. 298.

37. Sur l'Arcadia cf. Maria Teresa AcQuARo GRAzIosI, L'Arcadia. Trecento anni di storia, Roma, Palombi, 1991.

38. Cf. Carlo SALSOTTO, Le opere di Carlo Botta e la loro varia fortuna. Saggio di bibliografia critica con lettere inedite, Roma. F.lli Bocca, 1922. 
39. Traduction de Paul HAZARD, op. cit., p. 323, lettre du 10 mars 1810.

40. Cf. Paul VIGIER, « Diffusion d'une langue nationale et résistance des patois en France au XIX ${ }^{\mathrm{e}}$ siècle », Romantisme, 1979, n. 25-26, p. 191-208.

41. Michel De CERTEAU, Dominique Julia, Jacques ReVel, Une politique de la langue, la Révolution française et les patois, Paris, Gallimard, 1975.

42. Cf. Sven KöDEL, Die Enquête Coquebert de Montbret (1806-1812), Bamberg, University of Bamberg Press, 2014.

43. Rapport du Grand-Juge Ministre de la Justice, Section de législation. M. le Chevalier Faure, Rapporteur. $1^{\text {re }}$ Rédaction. $\mathrm{n}^{\mathrm{o}}$ d'enregistrement 32.806, Paris, De l'Imprimerie impérial, 5 mars 1812.

44. Ibid.

45. En ce qui concerne l'Allemagne, il est important de souligner les travaux de Claudie Paye sur la langue et la culture en Westphalie à l'époque napoléonienne : Claudie PAYE, Der französischen Sprache mächtig. Kommunikation im Spannungsfeld von Sprachen und Kulturen im Königreich Westphalen 1807-1813, Oldenbourg, München, Pariser Historische Studien, 2013 ; id., Cassel, prisme de l'identité westphalienne ou petit Paris au bord de la Fulda? Pratiques identitaires et frictions culturelles au royaume de Westphalie (1807-1813), Jacques-Olivier BOUDON, Gabriele B. CLEMENS (dir.), Erbfeinde im Empire? Franzosen und Deutsche im Zeitalter Napoleons, Ostfildern, Thorbecke, 2016, p. 33-50.

46. Fondamental pour l'étude de cette institution: G. TOMASI STUSSI, « Per la storia dell'Accademia Imperiale di Pisa (1810-1814)», Critica Storica, Firenze, Olschki, 1983, XX, n.1, p. 61-120.

47. Romano Paolo coppinI, Alessandro tosi, Alessandro volPI (éd.) L'Università di Napoleone. La riforma del sapere a Pisa, Pise, Edizioni Plus - Università di Pisa, 2004.

48. $\mathrm{AN}, \mathrm{F}^{17} 1602$; on utilise ici la version du rapport analysée et partiellement transcrite par Paolo Alvazzi Del Frate, Università napoleoniche negli Stati Romani. Il Rapport di Giovanni Ferri de SaintCostant sull'istruzione pubblica (1812), Roma, Viella, 1995.

49. L'entreprise au service de la Grande-Duchesse Elisa est un sujet qui n'a pas encore été étudié. Les archives, cependant, recèlent de nombreuses traces de la tournée de la compagnie dans les villes de Lucques, Pise, Livourne et Florence : cf. Archivio di Stato di Lucca (ASLu), Segreteria di Stato e di Gabinetto del Principato Lucchese, filza n. 218, Archivio di Stato di Pisa (ASP), Accademia dei Costanti, filza n. 19 et ASP, Teatro E. Rossi, Accademia dei Costanti, filza n. 9; Archivio di Stato di Livorno (ASLi), Accademia degli Avvalorati, filza n. 20 ; Archivio di Stato di Firenze (ASF), Accademia degli Intrepidi, Filza n. 116.

50. À propos de l'introduction du ballet, de l'opéra et de l'opera buffa à Rome: Archivio di Stato di Roma (ASRo), camerale II, VI teatri, n. 2126 e 2131; Archivio storico capitolino (ASC), arc XxVI, tomo 32, delibera del 21 aprile 1811. Sur le théâtre français dans le royaume de Naples cf. Valeria DE GREGORIO CIRILLO, I 'Comédiens français ordinaires du roi'. Gli spettacoli francesi al teatro del Fondo nel periodo napoleonico, Naples, Liguori, 2008.

51. À propos de l'influence française sur la langue administrative et juridique et les conséquences sur la langue italienne cf. Francesca FUSCO «Il linguaggio del diritto e della burocrazia nel XIX secolo tra aperture e istanze puristiche ", Italiano lingua due, n. 1, 2016, p. 246-268.

52. Jacques Boucher de Perthes raconte ce que devait être la situation à l'époque. Le 26 mars 1810, il écrit au directeur général des douanes romaines, Jean-Baptiste Collin de Sussy, au sujet de la composition des bureaux de la douane de Foligno : "Le personnel de ma division semble avoir été recruté sous la tour de Babel: Provençaux, Corses, Piémontais, Génois, Toscans, Romains, etc. ", Jacques BOUCHER DE PERTHES, Sous dix rois. Souvenirs de 1791 á 1860, vol. II, Paris, Dumulin, 1863, p. 39.

53. Il y aurait beaucoup d'exemples d'archives et documentaires, alors veuillez-vous référer au travail de Paul HAZARD, op. cit., paragraphes III et IV, chapitre I, livre II, p. 192-218.

54. Archivio di Stato di Genova (ASG), Prefettura francese, n. 165. 
55. Ivi, 2 juillet 1807.

56. Son Recueil raisonné des principales fonctions, devoirs et attributions des administrateurs des Comunes et des hospices, etc., entièrement en français est imprimé à Vercelli en 1806. Ce travail est cité dans Catalogo metodico della biblioteca del Ministero dell'Interno con indice alfabetico degli autori, Rome, Regia tipografia, 1872, p. 83. L'éditeur de l'œuvre de Crivelli étant un certain Felice Cerreti, imprimeur de la préfecture, on peut supposer que son travail a été soutenu par le gouvernement local.

57. Quelques exemples : ASP, Sottoprefettura di Pisa, busta n. 32, 16 mai 1808. ASLi, Prefettura del Mediterraneo, busta n. 19, 11 mai 1808. Ivi, 15 juin 1808, 25 juin 1808, 28 juin 1808, 29 juin 1808, 2 juillet 1808 e 6 juillet 1808 .

58. Un exemple de lettres adressées au sous-préfet de Pise Giovan Francesco Mastiani : ASP, Sottoprefettura di Pisa, busta n. 32, 26 avril 1809 et 27 avril 1809.

59. Cf. ASP, Sottoprefettura di Pisa, filza n.83.

60. En ce sens, il est intéressant d'analyser l'exemple d'un autre préfet, celui du département de Rome, Camille de Tournon, qui, comme Capelle, pouvait envoyer le même jour deux lettres au même destinataire, l'une en français et l'autre en italien (par exemple deux lettres à De Gerando datées du 17 janvier 1810, cf. AN F1 ${ }^{\mathrm{E}}$ 93). Dans certains cas, l'explication pourrait être que le fonctionnaire utilisait le français lorsqu'il écrivait lui-même la lettre pour les questions plus délicates, alors que pour les autres questions, il se fiait au secrétaire. Toutefois, il existe de nombreux exemples de lettres envoyées le même jour dans les deux langues sur le même sujet et au même destinataire. A cette pratique très particulière s'ajoute le fait que si, entre 1809 et le début de 1810, Tournon pouvait envoyer des lettres en français aux interlocuteurs français et italiens (ivi, 26 décembre 1809, 25 janvier 1810, 19-20 février 1810, 14 mai 1810; ASRo, camerale III, n. 2131, 23 mars 1810) dans la seconde moitié de 1810, toutes ses lettres étaient apparemment rédigées en italien (Ivi, lettres du 9 juin, 24 aout, 8 décembre 1810; AN, F1 ${ }^{\mathrm{E}}$ 160, 18 mai, 5 novembre 7 décembre; ASRo, Buon governo, n. 128, lettres du 13 novembre, 19 novembre, 24 novembre, 17 décembre, 24 décembre 1810 ; ASRo, Miscellanea de governo francese, n. 29, lettre à Cesare Balbo del 24 juillet 1810.

61. Bollettino delle leggi del Regno d'Italia, Milano, Presso la stamperia Reale Veladini, 1805, p. 33, p. 54 et p. 91.

62. Correspondance de Napoleon $I^{\text {er }}$, Paris, Plon \& Dumaine, 1861, t. 10, n. 8850, 7 juin 1805, p. 490.

63. Voici l'exemple d'une lettre en français de Carrafelli adressée au préfet de Bologne Francesco Mosca dans Archivio di Stato di Bologna (ASB), Prefettura del dipartimento del Reno, Tit. 14 Legislazione 1809, 5 octobre1809 ("J'ai l'honneur de vous prévenir, Monsieur le préfet, que je me rends à Milan... »). Une grande partie de la correspondance française provient des armées, qui s'adressent souvent aux préfets des territoires dans lesquels elles servent. Autres exemples de Bologne: ASB, prefettura del dipartimento del Reno, anno 1806, tit. xxVI, le commandant de l'armée au préfet du Reno, 23 décembre 1806; ibid., anno 1808, tit xxVI, le commandant de l'armée au préfet du Reno, 16 avril 1808.

64. Dans les documents du Podestà de Venise, il se trouve une lettre autographe d'Eugène de Beauharnais écrite en italien en 1810. Elle montre que le vice-roi était soucieux de paraître souple aux yeux les administrateurs locaux et qu'il commençait à essayer d'écrire lui-même en italien: «Vi ringrazio, Signor Podestà di Venezia, delle felicitazioni direttemi in nome vostro ed in quello del Municipio, intornò [sic] alla nascità di moi [sic] figlio. Sono felice pella parte che voi prendete alla mia gioia » (Archivio storico comunale di Venezia, anno 1810, Spettacoli, s.d.).

65. Jacques BOUCHER DE PERTHES, op. cit., vol. I, p. 474.

66. Giuseppe BERNARDONI, Elenco di alcune parole oggidi frequentemente in uso le quali non sono $n e$ ' vocabolari italiani, Milano, da' torchi di Gioanni Bernardoni, 1812.

67. Ettore PASSERIN D'ENTREVes, La giovinezza di Cesare Balbo, Firenze, Le Monnier, 1940. 
68. Cesare BALBo, Lettere di Carlo Vidua pubblicate da Cesare Balbo, Turin, Giuseppe Pomba, 1834.

69. Ibid., p. 174.

70. Ettore PASSERIN D'ENTREVES, op. cit., p.18.

71. Carlo viduA, Dello stato delle cognizioni in Italia. Discorso del Conte Carlo Vidua, Turin, Giuseppe Pomba, 1834. Ce travail a été publié en 1834, mais il était déjà achevé en 1813, quand Carlo Vidua avait laissé les aspirations littéraires pour les voyages qui lui conduiraient à l'Indonésie où il est mort de brûlures rapportées par l'éruption d'un volcan sur l'île de Celebes, aujourd'hui Sulawesi.

72. Cesare BALBO, op. cit., p. 297.

73. Ibid.

74. Antonio CESARI, Dissertazione sopra lo stato presente della lingua italiana, Verone, Ramanzani, 1810.

75. Maurizio VITALE, «Purista» $e$ «Purismo». Storia di parole e motivi della loro fortuna, dans L'oro nella lingua. Contributi per una storia del tradizionalismo e del purismo italiano, Milan-Naples, Ricciardi, 1986, p. 3-37.

76. Fabrizio FRANCESCHINI, op. cit., p. 305-310.

77. Rita LIBRANDI, « Ancora su Giuseppe Bernardoni, corrispondente di Monti, librettista e purista per caso ", dans Lingua e Stile, Rivista di storia della lingua italiana, n. 2, 2014, p. 237-266.

78. Alessandro MANZONI, «Il sistema del Padre Cesari» dans Opere inedite o rare di Alessandro Manzoni, Milan, Fratelli Rechiedei, 1898, p. 105-183.

79. Carteggio Alessandro Manzoni-Claude Fauriel, lettre $n^{\circ} 67$ (en français), Milan, Centro Nazionale di Studi manzoniani, 2000, p. 109.

80. Pour une étude approfondie de l'élite culturelle italienne comme moyene de continuité entre la phase révolutionnaire et la phase napoléonienne en Italie, et comment cette continuité contribue à nourrir la conscience nationale italienne Cf. Umberto CARPI, Patrioti e napoleonici. Alle origini dell'identità nazionale, Pise, Edizioni della Normale di Pisa, 2013.

81. Vincenzo MONTI, Proposta di alcune correzioni e aggiunte al Vocabolario della Crusca, Milan, Fontana, 1817. Pour une analyse du travail de Monti et de sa Proposta cf. Andrea DARDI, Gli scritti di Vincenzo Monti sulla lingua italiana, Firenze, Olschki, 1990.

82. Maurizio DARDANo, La lingua della nazione, Roma-Bari, Laterza, 2011.

\section{RÉSUMÉS}

Le travail de Paul Hazard sur le lien entre la Révolution française et les lettres italiennes n'a pas eu la postérité académique qu'il méritait. A cet égard, il est utile d'analyser les positions des intellectuels italiens de l'époque napoléonienne par rapport à l'occupation française de la péninsule italienne. La présence physique des Français dans l'administration et la société civile a également déclenché un changement dans la langue italienne. Les politiques linguistiques napoléoniennes, quoique pas uniformes dans tous les départements italiens, ont en outre joué un rôle dans l'évolution du débat intellectuel sur la langue italienne.

Paul Hazard's work on the link between the French Revolution and Italian letters did not get the academic development it deserved. In this respect, it is useful to analyse the debate of Italian intellectuals of the time in relation to the French occupation of the Italian peninsula during the 
Napoleonic period. The physical presence of the French in the administration and civil society had also triggered a change in the Italian language. Napoleonic linguistic policies, which were not uniform in all Italian departments, have also played a role in changing the intellectual debate on the Italian language.

INDEX

Mots-clés : langue italienne, Premier Empire, Napoléon, controverse linguistique.

Keywords : Italian language, First Empire, Napoleon, linguistic issue.

\section{AUTEUR}

ELISA BACCINI

Université de Padoue 